\title{
Differential Quadrature and Differential
}

\section{Transformation Methods in Buckling Analysis of Nanobeams}

https://doi.org/10.1515/cls-2019-0006

Received Sep 06, 2018; accepted Dec 03, 2018

\begin{abstract}
In this paper, two computationally efficient techniques viz. Differential Quadrature Method (DQM) and Differential Transformation Method (DTM) have been used for buckling analysis of Euler-Bernoulli nanobeam incorporation with the nonlocal theory of Eringen. Complete procedures of both the methods along with their mathematical formulations are discussed, and MATLAB codes have been developed for both the methods to handle the boundary conditions. Various classical boundary conditions such as SS, CS, and CC have been considered for investigation. A comparative study for the convergence of DQM and DTM approaches are carried out, and the obtained results are also illustrated to demonstrate the effects of the nonlocal parameter, aspect ratio $(\mathrm{L} / \mathrm{h})$ and the boundary condition on the critical buckling load parameter.
\end{abstract}

Keywords: Buckling; Nanobeam; DQM; DTM

\section{Introduction}

Implementations of nanomaterials are increasing significantly in various field of engineering and nanotechnology because of their peculiar properties [1]. Due to these properties, nanomaterials are considered as the most suitable material in various nano-electromechanical systems. These materials are mainly nanowires, nanoparticles, nanoribbons, nanotubes, and nano-electrical systems include nanoprobe, nanotube resonators [2], nanoactuators [3], and nanosensors, etc. One may find several applications of CNT reinforced structure in the literature [410]. Nanostructural elements may buckle when compres-

\footnotetext{
*Corresponding Author: S. Chakraverty: Department of Mathematics, National Institute of Technology, Rourkela-769008, India; Email: sne_chak@yahoo.com

Subrat Kumar Jena: Department of Mathematics, National Institute of Technology, Rourkela-769008, India; Email: sjena430@gmail.com
}

sive in-plane loads are applied on it, and surface stress itself has the property to induce buckling of a nanobeam with large deformation. So, it is very essential to determine the buckling characteristics of the nanobeams for the proper design of nanobeams. To the best of the present author's knowledge, the article provides first time a comparatively study for computing critical buckling parameters by using DQM, and DTM approaches based on EulerBernoulli beam theory. Accordingly, literature related to such problems using various methods have also been described in the next section.

\subsection{Development of DQM}

Bellman and Casti [11] for the first time introduced an efficient method namely Differential quadrature method (DQM). One may use this technique for solving linear and nonlinear problems arising from many physical model of science and technology. The main idea of this technique is that derivatives of any function at a particular point can be approximated by a linear combination of the functional values within a closed domain. Bert et al. [12] for the first time adopted this procedure to structural problems. After that, this technique becomes very popular among the researchers for solving numerous complicated problem associated with structural dynamics. Different authors tried to develop many approaches to handle boundary conditions smoothly. In this regards, Jang et al. [13] proposed $\delta$ technique to implement boundary conditions. In this approach [14], one boundary condition is used at the edge point while other is implemented at a distance $\delta$ from the edge point. But, this approach has two downsides. The use of boundary condition at the $\delta$ is not always exact which makes the solution less appropriate and more accurate numerical solutions can be obtained for the smaller value of $\delta$. Secondly, reducing the value of $\delta$ makes the solutions very unstable and it oscillates because of illconditioned weighting coefficient matrices. This is why the $\delta$ approach is significant to handel clamped end only. In order to eliminate this downside, Bert further came with

¿ Open Access. (c) 2019 S. K. Jena and S. Chakraverty, published by De Gruyter. 
a new approach for the implementation of boundary conditions which are available in the literature [15-19]. The main change in this approach is that only one boundary condition is used in one edge, and other edge is converted into the a matrices namely weighting coefficient matrices. Khaniki and Hosseini-Hashemi [20] uesd generalized differential quadrature method to analyze buckling behaviour of tapered nanobeams. One may also refer [21] where using the generalized differential quadrature method is used to study buckling analysis of nonuniform beams. Khaniki and Hosseini-Hashemi [22] investigated vibration characteristics of nonuniform nonlocal beams using nonlocal strain gradient theory. Tornabene et al. [23] extensively surveyed finite element method based differential quadrature method.

\subsection{Development of DTM}

DTM is a semi analytical-numerical technique which is mainly based on the idea of Taylor series expansion. Taylor series expansion method requires the computation of higher order derivatives which is not possible always where as differential transform method is based on iterative procedures. Zhou [24] proposed this technique in 1986 for solving linear and nonlinear initial value problems associated with electrical circuits. Chen and Ho [25] used this method for solving partial differential equations, and Ayaz $[26,27]$ solved initial value problem for partial differential equations by using two and three-dimensional DTM. Arikoglu and Ozkol [28] used this method to study integrodifferential equations. Further DTM technique has also been implemented to study rotating beam problems which are found in the literature [29-31]. Pradhan and Reddy [32] investigated buckling analysis of single-walled carbon nanotube on Winkler foundation using DTM. Nonlocal vibration analysis of functionally graded nanobeams was investigated by Ebrahimi et al. [33] using DTM. Also one can see [34] for flexural vibration of size-dependent functionally graded nanobeams is analyzed by differential transform method.

\section{Problem Formulation}

This investigation has been carried out on the EulerBernoulli beam in conjunction with Eringen [35] nonlocal theory of elasticity. From Hamilton's principle, the equation of motion of Euler-Bernoulli nanobeam for buckling can be written as [36]

$$
\frac{d^{2} M}{d x^{2}}=\bar{N} \frac{d^{2} y}{d x^{2}}
$$

For a one dimensional elastic material, nonlocal elasticity theory of Eringen [35] may be rewritten as

$$
\left(1-\left(e_{0} a\right)^{2} \frac{\partial^{2}}{\partial x^{2}}\right) \sigma_{x x}=E \varepsilon_{x x}
$$

Multiplying Eq. (2) by $z d A$ and integrating over $A$, the nonlocal constitutive relation for EBT may be expressed as

$$
M-\mu \frac{d^{2} M}{d x^{2}}=-E I \frac{d^{2} y}{d x^{2}}
$$

On simplifying and using Eq. (1) in Eq. (3), we may get

$$
M=-E I \frac{d^{2} y}{d x^{2}}+\mu\left(\bar{N} \frac{d^{2} y}{d x^{2}}\right)
$$

Plugging Eq. (4) in Eq. (1), we may have

$$
\frac{d^{2}}{d x^{2}}\left\{-E I \frac{d^{2} y}{d x^{2}}+\mu\left(\bar{N} \frac{d^{2} y}{d x^{2}}\right)\right\}=\bar{N} \frac{d^{2} y}{d x^{2}}
$$

After expansion, governing equation in terms of displacement is given as below.

$$
-E I \frac{d^{4} y}{d x^{4}}+\mu \bar{N} \frac{d^{4} y}{d x^{4}}-\bar{N} \frac{d^{2} y}{d x^{2}}=0,
$$

where $y$ is the transverse displacement, $E I$ is the bending rigidity, $\bar{N}$ is the applied axial compressive force and $\mu=$ $\left(e_{0} a\right)^{2}$ is the nonlocal parameter.

\section{Non-dimensional form of governing equation}

Following non-dimensional terms have taken for the present study:

$$
X=\frac{x}{L}, Y=\frac{y}{L}, \alpha=\frac{e_{0} a}{L} \text { and } \bar{N}^{0}=\frac{\bar{N} L^{2}}{E I} .
$$

By using the above-nondimensionalized forms, governing differential equation of Euler-Bernoulli beam theory is given by

$$
\frac{d^{4} Y}{d X^{4}}=\bar{N}^{0}\left(\alpha^{2} \frac{d^{4} Y}{d X^{4}}-\frac{d^{2} Y}{d X^{2}}\right) .
$$

Based on the above equation, solution of Euler-Bernoulli beam has obtained using Differential Quadrature Method (DQM) and Differential Transformation Method (DTM). For the sake of completeness, a brief review of DQM and DTM is presented here. 


\section{Numerical formulations}

In this section numerical procedures of DQM and DTM have been presented below to obtain the generalized eigenvalue problem for buckling analysis of EulerBernoulli nanobeam.

\subsection{Differential Quadrature Method (DQM)}

The derivatives of any function $Y(X)$ at a given point $i$ can be approximated as

$$
\begin{aligned}
Y_{i}^{\prime} & =\sum_{j=1}^{N} a_{i j} Y_{j} \\
Y_{i}^{\prime \prime} & =\sum_{j=1}^{N} b_{i j} Y_{j} \\
Y_{i}^{\prime \prime \prime} & =\sum_{j=1}^{N} c_{i j} Y_{j} \\
Y_{i}{ }^{I V} & =\sum_{j=1}^{N} d_{i j} Y_{j}
\end{aligned}
$$

where $i=1,2, \ldots, N$ and $N$ is the number of discrete grid points.

Here $a_{i j}, b_{i j}, c_{i j}$ and $d_{i j}$ are the matrices associated with the first, second, third and fourth derivatives respectively namely weighting coefficients matrices.

\subsubsection{Determination of weighting coefficients}

Computation of the weighting coefficient matrix $a=\left(a_{i j}\right)$ is the key step in the DQ method. In the present investigation, we have used Quan and Chang's [37] approach to compute weighting coefficients $a_{i j}$. As per this approach, matrix $a=\left(a_{i j}\right)$ may be computed by the following procedure.

For $i \neq j$

$$
a_{i j}=\frac{1}{X_{j}-X_{i}} \prod_{\substack{k \neq i \\ k \neq j \\ k=1}}^{N} \frac{X_{i}-X_{k}}{X_{j}-X_{k}} \quad i=1,2, \ldots, N j=1,2, \ldots, N
$$

\section{Simply supported- Simply supported}

for $i=j$

$$
a_{i i}=\sum_{\substack{k \neq i \\ k=1}}^{N} \frac{1}{X_{i}-X_{k}} \quad i=1,2, \ldots, N
$$
multiplication as follows
These $X_{i}^{\prime} s$ are Chebyshev-Gauss-Lobatto grid points which are given as below.

$$
X_{i}=\frac{1}{2}\left[1-\cos \left(\frac{i-1}{N-1} \cdot \pi\right)\right] .
$$

Once weighting coefficients associated with first-order derivatives are computed, weighting coefficients of higher order derivatives can be obtained easily by simple matrix

$$
\begin{gathered}
b=b_{i j}=\sum_{k=1}^{N} a_{i k} a_{k j} \\
c=c_{i j}=\sum_{k=1}^{N} a_{i k} b_{k j} \\
d=d_{i j}=\sum_{k=1}^{N} a_{i k} c_{k j}=\sum_{k=1}^{N} b_{i k} b_{k j} .
\end{gathered}
$$

\subsubsection{Application of boundary conditions}

Let us now denote

$$
\begin{aligned}
a & =\left[\begin{array}{ccccc}
a_{11} & a_{12} & \cdots & a_{1, N-1} & a_{1, N} \\
a_{21} & a_{22} & \cdots & a_{2, N-1} & a_{2, N} \\
\vdots & \vdots & & \vdots & \vdots \\
a_{N 1} & a_{N 2} & \cdots & a_{N, N-1} & a_{N, N}
\end{array}\right] \\
\bar{a} & =\left[\begin{array}{ccccc}
0 & a_{12} & \cdots & a_{1, N-1} & 0 \\
0 & a_{22} & \cdots & a_{2, N-1} & 0 \\
\vdots & \vdots & & \vdots & \vdots \\
0 & a_{N 2} & \cdots & a_{N, N-1} & 0
\end{array}\right] \\
\bar{a}_{1} & =\left[\begin{array}{cccc}
0 & a_{1,2} & \cdots & a_{1, N} \\
0 & a_{2,2} & \cdots & a_{2, N} \\
\cdots & \cdots & \cdots & \cdots \\
0 & a_{N, 2} & \cdots & a_{N, N}
\end{array}\right] \\
\bar{a}_{2} & =\left[\begin{array}{ccccc}
a_{1,1} & a_{1,2} & \cdots & a_{1, N-1} & 0 \\
a_{2,1} & a_{2,2} & \cdots & a_{2, N-1} & 0 \\
\cdots & \cdots & \cdots & \cdots & \cdots \\
a_{N, 1} & a_{N, 2} & \cdots & a_{N-1, N-1} & 0
\end{array}\right] .
\end{aligned}
$$

$$
\begin{aligned}
\left\{Y^{\prime}\right\} & =[\bar{a}]\{Y\} \\
\left\{Y^{\prime \prime}\right\} & =[a][\bar{a}]\{Y\}=[\bar{b}]\{Y\} \\
\left\{Y^{\prime \prime \prime}\right\} & =[\bar{a}]\left\{Y^{\prime \prime}\right\}=[\bar{a}][\bar{b}]\{Y\}=[\bar{c}]\{Y\} \\
\left\{Y^{I V}\right\} & =[a]\left\{Y^{\prime \prime \prime}\right\}=[a][\bar{c}]\{Y\}=[\bar{b}][\bar{b}]\{Y\}=[\bar{d}]\{Y\}
\end{aligned}
$$




\section{Clamped-simply supported:}

$$
\begin{aligned}
\left\{Y^{\prime}\right\} & =[\bar{a}]\{Y\} \\
\left\{Y^{\prime \prime}\right\} & =\left[\bar{a}_{1}\right]\left\{Y^{\prime}\right\}=\left[\bar{a}_{1}\right][\bar{a}]\{Y\}=[\bar{b}]\{Y\} \\
\left\{Y^{\prime \prime \prime}\right\} & =\left[\bar{a}_{2}\right]\left\{Y^{\prime \prime}\right\}=\left[\bar{a}_{2}\right][\bar{b}]\{Y\}=[\bar{c}]\{Y\} \\
\left\{Y^{I V}\right\} & =[a]\left\{Y^{\prime \prime \prime}\right\}=[a][\bar{c}]\{Y\}=[\bar{d}]\{Y\}
\end{aligned}
$$

\section{Clamped-Clamped:}

$$
\begin{aligned}
\left\{Y^{\prime}\right\} & =[\bar{a}]\{Y\} \\
\left\{Y^{\prime \prime}\right\} & =[\bar{a}]\left\{Y^{\prime}\right\}=[\bar{a}][\bar{a}]\{Y\}=[\bar{b}]\{Y\} \\
\left\{Y^{\prime \prime \prime}\right\} & =[a]\left\{Y^{\prime \prime}\right\}=[a][\bar{b}]\{Y\}=[\bar{c}]\{Y\} \\
\left\{Y^{I V}\right\} & =[a]\left\{Y^{\prime \prime \prime}\right\}=[a][\bar{c}]\{Y\}=[\bar{d}]\{Y\}
\end{aligned}
$$

Plugging the value of Eq. (8) into Eq. (7), one may obtain generalized eigenvalue problem as

$$
[S]\{Y\}=\bar{N}^{0}[T]\{Y\}
$$

where $S$ is the stiffness matrix and $T$ is the mass matrix.

\subsection{Differential transformation method (DTM)}

The differential transformation of an analytic function $f(x)$ in a domain $\mathrm{D}$ at a point $x=x_{0}$ can be expressed by a power series with center is at $x_{0}$ as given below.

$$
F(k)=\frac{1}{k !}\left(\frac{d^{k} f(x)}{d x^{k}}\right)_{x=x_{0}}
$$

where $f(x)$ is the function to be transformed and $F(k)$ is the transformed function. The inverse transformation is defined as

$$
f(x)=\sum_{k=0}^{\infty}\left(x-x_{0}\right)^{k} F(k) .
$$

Adding both the equations (15) and (16), we may have

$$
f(x)=\sum_{k=0}^{\infty} \frac{\left(x-x_{0}\right)^{k}}{k !}\left(\frac{d^{k} f(x)}{d x^{k}}\right)_{x=x_{0}}
$$

In actual applications, the function $f(x)$ is expressed by a finite series and equation (17) can be written as follows

$$
f(x)=\sum_{k=0}^{m} \frac{\left(x-x_{0}\right)^{k}}{k !}\left(\frac{d^{k} f(x)}{d x^{k}}\right)_{x=x_{0}}
$$

For basic operations of DTM on various function as well as boundary conditions, one may refer Özdemir and Kaya [31].
Table 1: Basic operations of DTM for the governing equations

\begin{tabular}{cc}
\hline Original function & Transformed function \\
\hline$f(x)=g(x) \pm h(x)$ & $F(k)=G(k) \pm H(k)$ \\
$f(x)=\lambda g(x)$ & $F(k)=\lambda G(k)$ \\
$f(x)=g(x) h(x)$ & $F(k)=\sum_{l=0}^{k} G(l) H(k-l)$ \\
$f(x)=\frac{d^{n} g(x)}{d x^{n}}$ & $F(k)=\frac{(k+n) !}{k !} G(k+n)$ \\
$f(x)=x^{n}$ & $F(k)=\delta(k-n)$ \\
\hline
\end{tabular}

\subsubsection{DTM formulation and solution procedure}

Using DTM and referring to Table 1, the dimensionless governing equation of motion (Eq. (7)) is transformed into the following equation

$$
\begin{aligned}
& (k+1)(k+2)(k+3)(k+4) \bar{Y}(k+4) \\
& =\bar{N}^{0}\left(\alpha^{2}(k+1)(k+2)(k+3)(k+4) \bar{Y}(k+4)\right. \\
& -(k+1)(k+2) \bar{Y}(k+2))
\end{aligned}
$$

If the above equation is rearranged, a recurrence relation can be obtained as

$$
\bar{Y}(k+4)=\frac{\bar{N}^{0} \bar{Y}(k+2)}{\left(\bar{N}^{0} \alpha^{2}-1\right)(k+3)(k+4)} .
$$

Then boundary conditions can be written as in Table 2 .

Let us now consider particular case, say, of a C-S beam, having the $x=0$ edge as clamped and $x=1$ edge as simply supported. By performing DTM to this boundary condition, with origin chosen at the clamped end, we may have

$$
\begin{aligned}
& \bar{Y}(0)=0 ; \\
& \bar{Y}(1)=0 .
\end{aligned}
$$

At the simply supported end, that is, at $x=1$, we also have

$$
\begin{aligned}
& \sum_{k=0}^{\infty} \bar{Y}(k)=0 ; \\
& \sum_{k=0}^{\infty} k(k-1) \bar{Y}(k)=0 .
\end{aligned}
$$

By using the clamped boundary condition that is Eq. (20) in recurrence relation Eq. (19), it can be seen that $\bar{Y}(k)$ is a linear function of $\bar{Y}(2)$. Now simply supported end boundary condition that is Eq. (21) can be written in matrix form as

$$
\left[\begin{array}{ll}
f_{11} & f_{12} \\
f_{21} & f_{22}
\end{array}\right]\left[\begin{array}{l}
\bar{Y}(2) \\
\bar{Y}(3)
\end{array}\right]=\left[\begin{array}{l}
0 \\
0
\end{array}\right]
$$


Table 2: Differential Transformation for SS, CF, CC and CS boundary condition

\begin{tabular}{cccc}
\hline SS & CF & CC & CS \\
\hline $\bar{Y}(0)=\bar{Y}(2)=0$ & $\bar{Y}(0)=\bar{Y}(1)=0$ & $\bar{Y}(0)=\bar{Y}(1)=0$ & $\bar{Y}(0)=\bar{Y}(1)=0$ \\
$\sum_{k=0}^{\infty} \bar{Y}(k)=0$ & $\sum_{k=0}^{\infty} k(k-1) \bar{Y}(k)=0$ & $\sum_{k=0}^{\infty} \bar{Y}(k)=0$ & $\sum_{k=0}^{\infty} \bar{Y}(k)=0$ \\
$\sum_{k=0}^{\infty} k(k-1) \bar{Y}(k)=0$ & $\sum_{k=0}^{\infty} k(k-1)(k-2) \bar{Y}(k)=0$ & $\sum_{k=0}^{\infty} k \bar{Y}(k)=0$ & $\sum_{k=0}^{\infty} k(k-1) \bar{Y}(k)=0$ \\
\hline
\end{tabular}
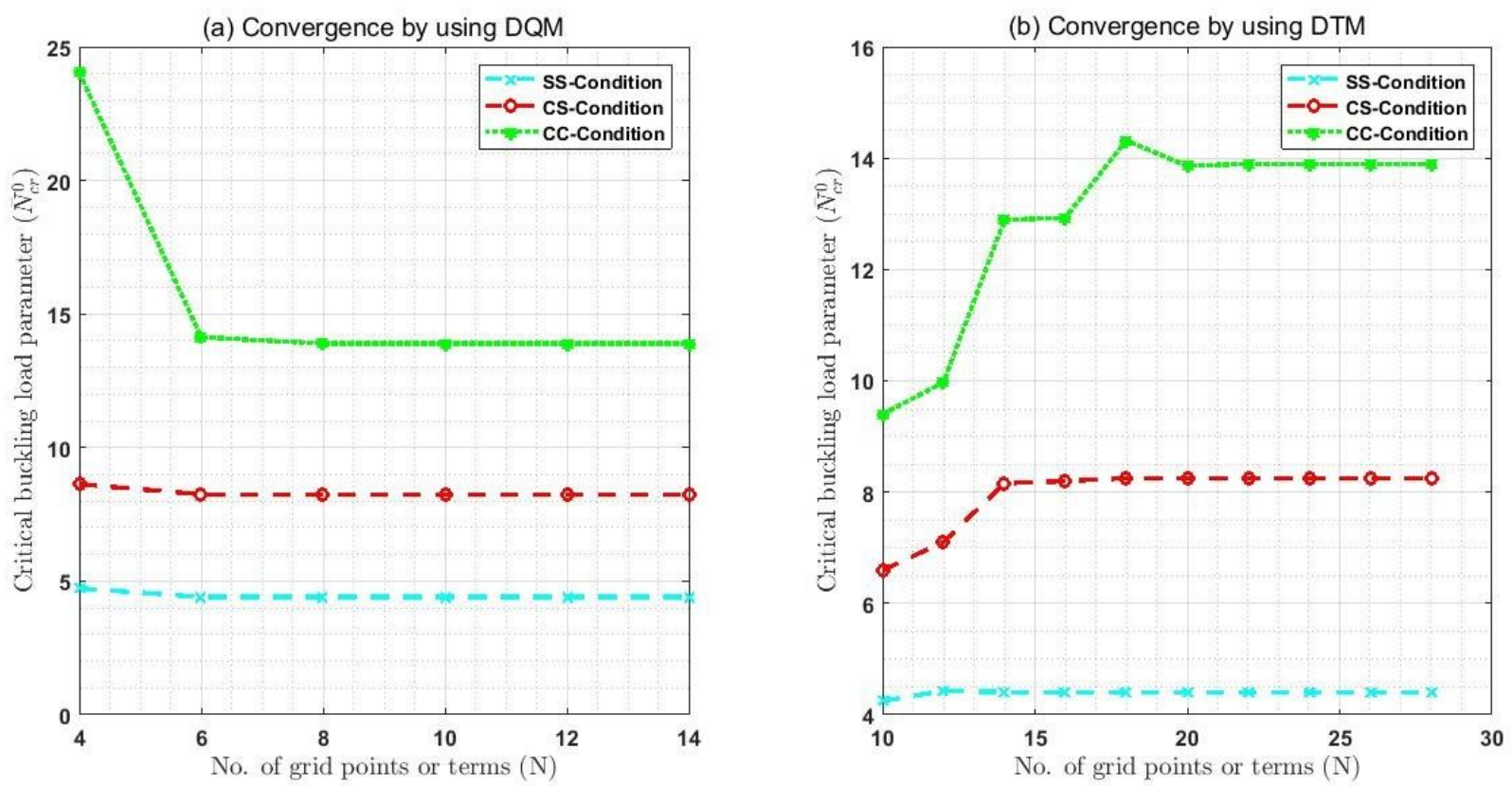

Figure 1: Critical buckling parameter Vs. number of terms (grid points) for both DQM and DTM

For $\bar{Y}(2) \neq 0, \bar{Y}(3) \neq 0$, the eigenvalue problem may be converted as

$$
\left|\begin{array}{ll}
f_{11} & f_{12} \\
f_{21} & f_{22}
\end{array}\right|=0
$$

Solving the above Eq. (23), we will get eigenvalue $\lambda=\lambda_{i}^{n}$ where $i=1,2$ and $\lambda_{i}^{n}$ is the $i^{t h}$ estimated eigenvalue corresponding to $n$. One may obtain the value of $n$ from the following identity;

$$
\left|\lambda_{i}^{n}-\lambda_{i}^{n-1}\right| \leq \varepsilon
$$

Where $\lambda_{i}^{n}$ is the estimated eigenvalue corresponding to $n$ and $\lambda_{i}^{n-1}$ is the $i^{\text {th }}$ estimated eigenvalue corresponding to $n-1$. We will get $i^{\text {th }}$ eigenvalue $\lambda_{i}$ after Eq. (24) is satisfied.

\section{Numerical results and discussions}

MATLAB program is developed to obtain the numerical results by solving Eq. (14) and Eq. (22). The DQM and DTM have been employed, and the boundary conditions are implemented. In this study following parameters are considered for the computation purpose: $\mathrm{E}=1 \mathrm{TPa}, \mathrm{L}=10 \mathrm{~nm}, \mathrm{~h}=$ $1 \mathrm{~nm}, \mathrm{~d}=1 \mathrm{~nm}$.

\subsection{Convergence of DQM and DTM approach}

In this subsection, a convergence study is carried out for both DQM and DTM approach. From table 3 and Figure 1, one may observe that critical buckling load parameters by DQM converge faster than DTM. Also one may notice that critical buckling load parameters of SS end converge faster in each method whereas it takes more number of terms in the case of CC end. Moreover, DQM takes 10 grid points for 
converging of all B.C.s whereas DTM requires 24 terms for the same. In this case, has been taken as $1 \mathrm{~nm}$ and $L / h=$ 10 .

Table 3: Convergence of both DQM and DTM approach

\begin{tabular}{cccc} 
(a) & Convergence of DQM $\left(L / h=10, e_{0} a=1\right)$ & \\
\hline & $\mathrm{SS}$ & $\mathrm{CS}$ & $\mathrm{CC}$ \\
\hline 4 & 4.7313 & 8.6307 & 24.0428 \\
6 & 4.4085 & 8.2487 & 14.1359 \\
8 & 4.4095 & 8.2460 & 13.8948 \\
10 & 4.4095 & 8.2461 & 13.8938 \\
12 & 4.4095 & 8.2461 & 13.8938 \\
14 & 4.4095 & 8.2461 & 13.8938
\end{tabular}

(b) Convergence of DTM $\left(L / d=10, e_{0} a=1\right)$

\begin{tabular}{cccc}
\hline$N$ & SS & CS & CC \\
\hline 10 & 4.2497 & 6.5945 & 9.3989 \\
12 & 4.4276 & 7.1035 & 9.9729 \\
14 & 4.4095 & 8.1586 & 12.8907 \\
16 & 4.4095 & 8.1966 & 12.9323 \\
18 & 4.4095 & 8.2506 & 14.3156 \\
20 & 4.4095 & 8.2458 & 13.8601 \\
22 & 4.4095 & 8.2461 & 13.8971 \\
24 & 4.4095 & 8.2461 & 13.8935 \\
26 & 4.4095 & 8.2461 & 13.8938 \\
28 & 4.4095 & 8.2461 & 13.8938 \\
\hline
\end{tabular}

\subsection{Validation}

Validations of both DQM and DTM approaches are accomplished by comparing the obtained results with that available in the literature [38]. For this purpose, the same parameters are used as in Ref. [38]. Comparison of the critical buckling load parameters for SS, CS and CC nanobeams have been depicted in Table 4 for different value of $\left(e_{0} a\right)$. In this case aspect ratio $(L / h)$ has been taken as 10 .

\subsection{Small scale effect}

The importance of the small scale coefficient is discussed in this subsection, Small-scale effect is mainly dealt with the response of buckling load ratio with scale coefficient $\left(e_{0} a\right)$. Buckling load ratio is defined as $\bar{N}_{c r}^{0}$ obtained using the nonlocal theory and $\bar{N}_{c r}^{0}$ obtained using local theory. This buckling load ratio is very essential to estimate the small scale effect on the buckling solution. Response of the buckling load ratio with scale coefficient $\left(e_{0} a\right)$ is depicted
Table 4: Comparisons of DQM and DTM with Ref. [38]

(a) Comparisons of DQM and DTM with Ref. [38] for SS Condition

\begin{tabular}{cccc}
\hline$e_{0} a$ & DQM & DTM & Ref. [38] \\
\hline 0 & 4.8447 & 4.8447 & 4.8447 \\
0.5 & 4.7281 & 4.7281 & 4.7281 \\
1.0 & 4.4095 & 4.4095 & 4.4095 \\
1.5 & 3.9644 & 3.9644 & 3.9644 \\
2.0 & 3.4735 & 3.4735 & 3.4735 \\
\hline
\end{tabular}

(b) Comparisons of DQM and DTM with Ref. [38] for CS Condition

\begin{tabular}{cccc}
\hline$e_{0} a$ & DQM & DTM & Ref. [38] \\
\hline 0 & 9.9155 & 9.9155 & 9.9155 \\
0.5 & 9.4349 & 9.4349 & 9.4349 \\
1.0 & 8.2461 & 8.2461 & 8.2461 \\
1.5 & 6.8151 & 6.8151 & 6.8151 \\
2.0 & 5.4830 & 5.4830 & 5.4830 \\
\hline
\end{tabular}

(c) Comparisons of DQM and DTM with Ref. [38] for CC Condition

\begin{tabular}{cccc}
\hline$e_{0} a$ & DQM & DTM & Ref. [38] \\
\hline 0 & 19.3790 & 19.3790 & 19.3790 \\
0.5 & 17.6381 & 17.6381 & 17.6381 \\
1.0 & 13.8939 & 13.8939 & 13.8939 \\
1.5 & 10.2630 & 10.2630 & 10.2630 \\
2.0 & 7.5137 & 7.5137 & 7.5137 \\
\hline
\end{tabular}

in Figure 2 and Table 5 for all the edge conditions viz. SS, $\mathrm{CS}$, and CC with $L / h=10$. One may also notice from the figure that the buckling load ratios are less than unity which implies that the application of the local beam model for the buckling analysis of carbon nanotubes would lead to overprediction of the buckling load if the small length scale effect between the individual carbon atoms is ignored. The buckling loads obtained by the nonlocal beam model is smaller than its local counterpart with increasing scale coefficient $e_{0} a$. That means the buckling load parameter obtained by the local beam theory is larger than that obtained by the nonlocal beam theory. So the presence of the nonlocal parameter in the constitutive equation is significant in the field of nanomechanics.

\subsection{Effect of boundary conditions}

Boundary conditions is essential in designing any engineering structures. It helps designers to acquire an idea without carrying out a much detailed investigation. Therefore, analysis of the boundary conditions is essential. In this subsection, the effect of the boundary condition on 
Table 5: Buckling load ratio for various scale coefficient

(a) Buckling load ratio for various scale coefficient (SS Condition)

\begin{tabular}{ccccccc}
\hline$e_{0} a$ & 0 & 0.2 & 0.4 & 0.6 & 0.8 & 1.0 \\
\hline DQM & 4.8447 & 4.8256 & 4.7694 & 4.6785 & 4.5568 & 4.4095 \\
DTM & 4.8447 & 4.8256 & 4.7694 & 4.6785 & 4.5568 & 4.4095 \\
Buckling load Ratio & 1 & 0.9960 & 0.9844 & 0.9656 & 0.9405 & 0.9101 \\
\hline
\end{tabular}

(b) Buckling load ratio for various scale coefficient (CS Condition)

\begin{tabular}{ccccccc}
\hline$e_{0} a$ & 0 & 0.2 & 0.4 & 0.6 & 0.8 & 1.0 \\
\hline DQM & 9.9155 & 9.8316 & 9.6009 & 9.2395 & 8.7769 & 8.2461 \\
DTM & 9.9155 & 9.8316 & 9.6009 & 9.2395 & 8.7769 & 8.2461 \\
Buckling load Ratio & 1 & 0.9915 & 0.9682 & 0.9318 & 0.8851 & 0.8316 \\
\hline
\end{tabular}

(c) Buckling load ratio for various scale coefficient (CC Condition)

\begin{tabular}{ccccccc}
\hline$e_{0} a$ & 0 & 0.2 & 0.4 & 0.6 & 0.8 & 1.0 \\
\hline DQM & 19.3790 & 19.0776 & 18.2275 & 16.9674 & 15.4701 & 13.8939 \\
DTM & 19.3790 & 19.0776 & 18.2275 & 16.9674 & 15.4701 & 13.8939 \\
Buckling load Ratio & 1 & 0.9844 & 0.9405 & 0.8755 & 0.7982 & 0.7169 \\
\hline
\end{tabular}

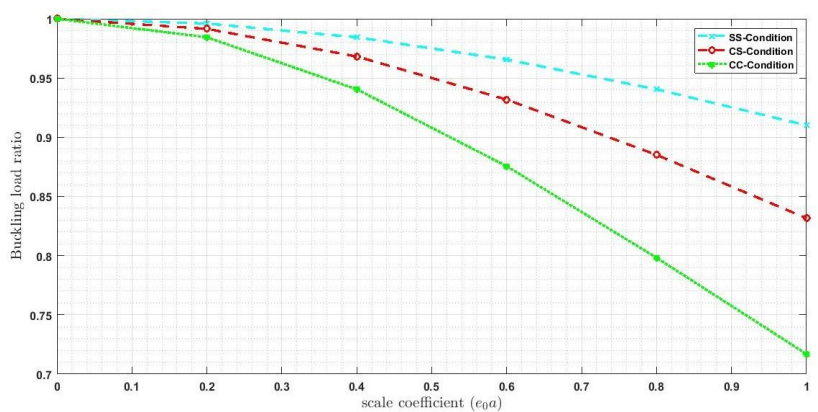

Figure 2: Buckling load ratio Vs. scale coefficient $\left(e_{0} a\right)$

the critical buckling load parameter is analyzed which is demonstrated in Figure 3. This variation of the critical buckling load parameter with the scale coefficient for different boundary conditions is considered with $\mathrm{L}=10$ $\mathrm{nm}$. One may observe from the figure that $\mathrm{CC}$ nanobeam possesses the highest critical buckling load parameter whereas SS nanobeams have the lowest critical buckling load parameter.

\subsection{Effects of the aspect ratio $(L / h)$}

Aspect ratio also plays a significant role in the design of engineering structures. To study the aspect ratio $(\mathrm{L} / \mathrm{h})$ on the critical buckling load parameter, the variation of the critical buckling load parameter with $\mathrm{L} / \mathrm{h}$ has been depicted in Figure 4. The results are shown for scale coefficients 1 $\mathrm{nm}$. It may be noted that the critical buckling load param-

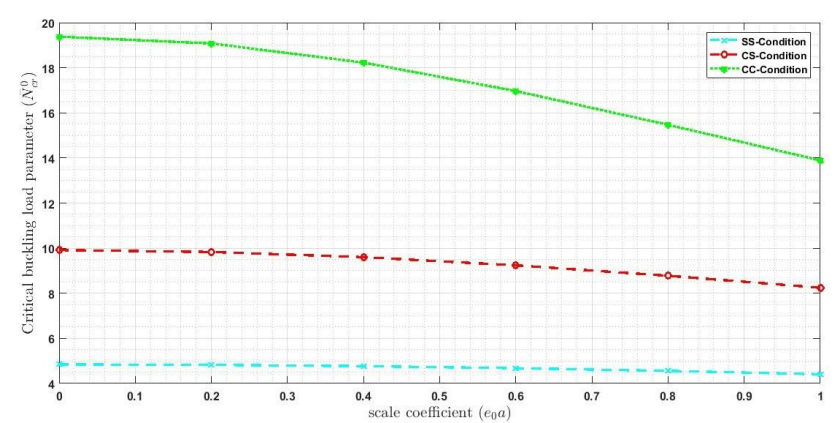

Figure 3: Critical buckling load parameter Vs. scale coefficient for different B.C.S

eter decreases with increase in $\mathrm{L} / \mathrm{h}$ which may be seen in Table 6 for the boundary conditions SS, CS, and CC.

Table 6: Critical buckling load Parameter for various $\mathrm{L} / \mathrm{h}$

\begin{tabular}{cccc}
\hline \multirow{2}{*}{$L / h$} & \multicolumn{3}{c}{ Critical Buckling Load Parameter $\left(\bar{N}_{c r}^{0}\right)$} \\
\cline { 2 - 4 } & SS & CS & CC \\
\hline 10 & 4.4095 & 8.2461 & 13.8939 \\
20 & 1.1820 & 2.3587 & 4.4095 \\
30 & 0.5324 & 1.0770 & 2.0627 \\
40 & 0.3009 & 0.6117 & 1.1820 \\
50 & 0.1930 & 0.3932 & 0.7631 \\
\hline
\end{tabular}




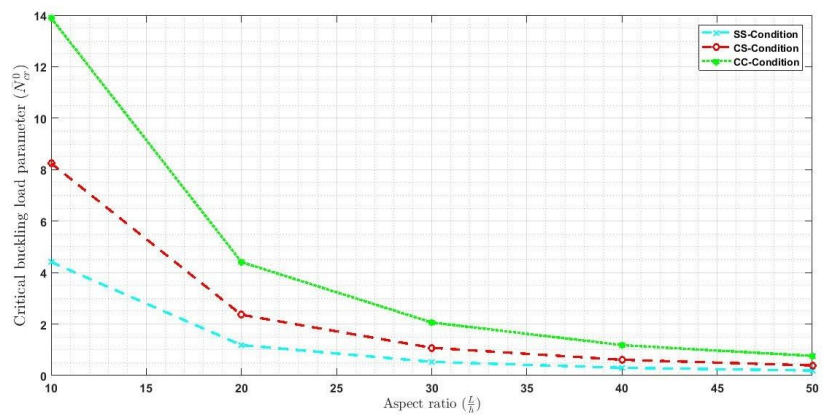

Figure 4: Critical buckling load parameter Vs. aspect ratio

\section{Concluding Remarks}

Buckling analysis of Euler-Bernoulli nanobeams based on nonlocal theory has been carried out by DQM and DTM. Convergence of DQM and DTM approaches are carried out, and it is observed that former one converges more rapidly than later one. Tabular and graphical results are explored to illustrate the response of the nonlocal parameter, $\mathrm{L} / \mathrm{h}$ and the boundary condition on the critical buckling load parameter. One may observe that the critical buckling load parameter decreases with increase in the nonlocal parameter. It may also be noticed that CC nanobeams have higher buckling loads than the other types of boundary conditions. Further, we can extend this approach to investigate critical buckling parameter of curved structures like vaults, domes, and barrels arising in various engineering problem by this two computationally efficient techniques viz. Differential Quadrature Method (DQM) and Differential Transformation Method (DTM) because of their high efficiency and accuracy.

Acknowledgement: The authors are thankful to Defence Research \& Development Organization(DRDO), Ministry of Defence, New Delhi, India (Sanction Code: DG/TM/ERIPR/GIA/17-18/0129/020) for the support and funding to carry out the present research work.

\section{References}

[1] Dai H, Hafner JH, Rinzler AG, Colbert DT, Smalley RE (1996) Nanotubes as nanoprobes in scanning probe microscopy. Nature 384:147-150

[2] Peng H, Chang C, Aloni S, Yuzvinsky T, Zettl A (2006) Ultrahigh frequency nanotube resonators. Phys. Rev. Lett. 97:087203(1-4)

[3] Dubey A, Sharma G, Mavroidis C, Tomassone M, Nikitczuk K, M. Yarmush (2004) Computational studies of viral protein nanoactuators. J. Comput. Theor. Nanosci.1:18-28
[4] Tornabene F, Fantuzzi N, Bacciocchi M, Viola E (2016) Effect of agglomeration on the natural frequencies of functionally graded carbon nanotube-reinforced laminated composite doubly-curved shells. Composites Part B 89:187-218.

[5] Fantuzzi N, Tornabene F, Bacciocchi M, Dimitri R (2017) Free vibration analysis of arbitrarily shaped Functionally Graded Carbon Nanotube-reinforced plates. Composites Part B 115:384408.

[6] Tornabene F, Fantuzzi N, Bacciocchi M (2017) Linear static response of nanocomposite plates and shells reinforced by agglomerated carbon nanotubes. Composites Part B 115:449-476.

[7] Bani'c D, Bacciocchi M, Tornabene F, Ferreira AJM (2017) Influence of Winkler-Pasternak Foundation on the Vibrational Behavior of Plates and Shells Reinforced by Agglomerated Carbon Nanotubes. Applied Sciences 7:1-55.

[8] Seidi J, Kamarian S (2017) Free vibrations of non-uniform $\mathrm{CNT} /$ fiber/polymer nanocomposite beams. Curved and Layered Structures 4:21-30.

[9] Zhong R, Wang Q, Tang J, Shuai C, Liang Q (2018) Vibration characteristics of functionally graded carbon nanotube reinforced composite rectangular plates on Pasternak foundation with arbitrary boundary conditions and internal line supports. Curved and Layered Structures 5:10-34.

[10] Pang F, Li H, Du Y, Shan Y, Ji F (2018) Free vibration of functionally graded carbon nanotube reinforced composite annular sector plate with general boundary supports. Curved and Layered Structures 5:49-67.

[11] Bellman R, Casti J (1971) Differential quadrature and long-term integration. J. Math. Anal. Appl. 34: 235-238

[12] Bert CW, Jang SK, Striz AG (1988) Two new approximate methods for analyzing free vibration of structural components. AIAA J. 26: 612-618

[13] Jang SK, Bert CW, Striz AG (1989) Application of differential quadrature to static analysis of structural components. Internat. J. Numer. Methods Engrg. 28:561-577

[14] Shu C (2000) Differential Quadrature and its Application in Engineering. Springer-Verlag, London

[15] Wang X, Bert CW (1993) A new approach in applying differential quadrature to static and free vibrational analyses of beams and plates. J. Sound Vib.162:566-572

[16] Bert CW, Wang X, Striz AG (1994) Static and free vibrational analysis of beams and plates by differential quadrature method. Acta Mech.102:11-24

[17] Wang X, Bert CW, Striz AG (1993) Differential quadrature analysis of deflection, buckling, and free vibration of beams and rectangular plates. Comput. \& Structures 48:473-479

[18] Bert CW, Wang X, Striz AG (1993) Differential quadrature for static and free vibration analyses of anisotropic plates. Internat. J. Solids Structures 30:1737-1744

[19] Bert CW, Malik M (1996) The differential quadrature method for irregular domains and application to plate vibration. Int. J. Mech. Sci. 38:589-606

[20] Khaniki HB, Hashemi SH (2017) Buckling analysis of tapered nanobeams using nonlocal strain gradient theory and a generalized differential quadrature method. Materials Research Express 4: 065003(1-22).

[21] Khaniki HB, Hashemi SH, Nezamabadi A (2017) Buckling analysis of nonuniform nonlocal strain gradient beams using generalized differential quadrature method. Alexandria Engineering Journal, doi: 10.1016/j.aej.2017.06.001. 
[22] Khaniki HB, Hashemi SH (2017) Dynamic transverse vibration characteristics of nonuniform nonlocal strain gradient beams using the generalized differential quadrature method. The European Physical Journal Plus 132: 500(1-15).

[23] Tornabene F, Fantuzzi N, Ubertini F, Viola E (2015) Strong Formulation Finite Element Method Based on Differential Quadrature: A Survey. Appl. Mech. Rev. 67:020801 (1-55).

[24] Zhou JK (1986) Differential Transformation and its Application for Electrical Circuits. Huazhong University Press 1:96-102

[25] Chen CK, Ho SH (1999) Solving partial differential equations by two-dimensional differential transform method. Appl. Math.Comput.106:171-179.

[26] Ayaz F (2003) On the two-dimensional differential transform method. Appl. Math. Comput.143:361-374

[27] Ayaz F (2004) Solutions of the system of differential equations by differential transform method. Appl. Math. Comput.147:547567

[28] Arıkoglu A, Özkol I (2005) Solution of boundary value problems for integro-differential equations by using differential transform method. Appl. Math. Comput.168:1145-1158

[29] Özdemir Ö, Kaya MO (2006) Flapwise bending vibration analysis of a rotating tapered cantilever Bernoulli-Euler beam by differential transform method. J. Sound Vib. 289:413-420

[30] Kaya MO (2006) Free vibration analysis of rotating Timoshenko beam by differential transform method. Aircr. Eng. AerospTechnol.78:194-203
[31] Özdemir Ö, Kaya MO (2006) Flapwise bending vibration analysis of double tapered rotating Euler-Bernoulli beam by using the differential transform method. Meccanica 41:661-670

[32] Pradhan SC, Reddy GK (2011) Buckling analysis of single walled carbon nanotube on Winkler foundation using nonlocal elasticity theory and DTM. Computational Materials Science 50:10521056.

[33] Ebrahimi F, Ghadiri M, Salari E, Hoseini SAH, Shaghaghi GR (2015) Application of the differential transformation method for nonlocal vibration analysis of functionally graded nanobeams. Journal of Mechanical Science and Technology 29:1207-1215.

[34] Ebrahimi F, Salari E (2015). Size-dependent free flexural vibrational behavior of functionally graded nanobeams using semianalytical differential transform method. Composites Part B: Engineering 79:156-169.

[35] Eringen A C (1972) International Journal of Engineering Science 1:157-308

[36] Reddy JN (2007) Nonlocal theories for bending, buckling and vibration of beams. International Journal of Engineering Science 45: 288-307

[37] Quan J, Chang C (1989) New insights in solving distributed system equations by the quadrature method-I. Analysis, Comput. Chem. Eng.13:779-788

[38] Wang CM, Zhang YY, Ramesh SS, Kitipornchai S (2006) Buckling analysis of micro- and nano-rods/tubes based on nonlocal Timoshenko beam theory. J. Phys.D: Appl. Phys. 39: 3904 